\title{
Managing Smart City Power Network by Shifting Electricity Consumers Demand
}

\author{
Cátia Silva ${ }^{1}$, Pedro Faria ${ }^{1}$ and Zita Vale ${ }^{2}$ \\ ${ }^{1}$ GECAD - Research Group on Intelligent Engineering and Computing for Advanced Innova- \\ tion and Development, Porto, Portugal \\ ${ }^{2}$ Polytechnic of Porto, Porto, Portugal \\ crcds@isep.ipp.pt; pnf@isep.ipp.pt; zav@isep.ipp.pt
}

\begin{abstract}
Demand Response (DR) concept, introduced by the Smart Grid paradigm, is presented as one of the main solutions to mitigate the effects of the intermittency of Distributed Generation sources in the network. With this, the consumer's role in the energy market is empowered and their flexibility is crucial. The technology advances allow bidirectional communication providing signals to the active consumers, given by the entity manager, participating in DR events to alleviate problems in the grid. The authors proposed a methodology, resorting to load shifting, to lessen voltage limit violations in some points of the grid. After the detention, the DR event is triggered and the small resources are scheduled, requesting a reduction to the active consumers. The authors believe that uncertainty in the response must be considered so, the willingness and availability from the active community are contemplated on the problem. The results show the necessity of pondering consumer behavior.
\end{abstract}

Keywords: Demand Response, Load Shifting, Uncertainty, Remuneration.

\section{Introduction}

The world's population is increasing, and the concept of smart cities was born to provide the answers for the problems that consequently arise - for instance, unsustainable energy and water use, pollution, or even environmental degradation. It is expected that, by implementing information and communication technologies, some of the consequences will be mitigated and will help to properly manage the communities [1]. In this way, the consumer's role in the energy market is changing. Until now, low or no information was given to the end-users regarding the transactions in the market. With the increasing penetration of the Distributed Generation (DG) in the grid, namely solar and wind, the prior paradigm is no longer valid - the generation follows and satisfies the consumption needs [2]. Instead, the demand side must adapt their needs to the available production since the behavior from the renewable-based technologies is intermittent [3].

The Demand Side Management solutions are considered, by the literature as the solution to avoid costly investments in the system restructure as well as aid on alleviate the problems that will arise from demand peaks [4]. Thus, Demand Response (DR) 
programs should be implemented with the help of bidirectional communication and Advanced Metering Infrastructures, including smart meters and smart appliances [5], [6]. Having information and understanding the consumer load pattern, aid the entity manager in giving the right signals to change the consumption to alleviate any difficulties found in the grid, ensuring the security and reliability of the system [7], [8]. So, the role of small consumers in the energy market is changing. Yet, according to Iliana Ilieva, et al. [9] it will take time, education, and resources until taking rational decisions in the market transactions. However, the actual business models do not include or can deal with the uncertainty associated with these new resources. The unpredictable behavior, from both consumers and DG units, increase the complexity of managing the network. An entity was created to be responsible for the transactions in local communities with active players - the Aggregator. It is believed that developing smart buildings and smart homes, giving the right tools for the active consumers is a great step forward to the implementation in the real world of DR programs. However, other issues resulting from the use of such technologies, for instance, the large amounts of data, concerns regarding data security, safety, and privacy. The designed method to successfully implement DR in the real market should be able to manage these matters - artificial intelligence and machine learning are widely used in the treatment of big data.

Many models in the literature were created finding solutions to optimally manage and to aid and decrease the difficulty when dealing with the new players. Shaghayegh Zalzar and Ettore Francesco Bompard [10] modeled the demand flexibility using load shifting focusing and proposing an incentive-based settlement mechanism. The results were withdrawn, with high penetration of wind energy, indicate that monetary incentives are crucial for active consumer participation - without them, it is not profitable to provide such flexibility. Özge Okur et al. [11] evaluated the impact of DR for internal balancing to reduce the individual imbalances of an Aggregator regarding uncertain DG resorting to Model Predictive Control, reducing up to $30 \%$ in one of the case studies. However, the remuneration of the active consumers is not considered in their work.

The authors proposed a method to properly manage an active community in a smart city by developing a tool to aid the Aggregator on the complex task of handling uncertain resources. Being a continuity from other works [12], [13], this paper it is presented a resolution for a voltage limit violation mitigated by triggering a DR event. After the detection of the problem, a DR event is triggered and flexibility from the active consumers is requested after the scheduling of the resources. Load shifting is applied, and the consumption is moved to another period. The authors consider the consumer perspective a crucial matter in developing DR solutions [14]. In this way, the availability, and the willingness to contribute to such programs must be deemed. In this way, the case study is based on a smart city and how the Aggregator should manage the small resources associated, which can be a complex task due to the uncertainty of the active consumer response and the intermittent behavior of the DG resources.

The present paper is structured into five main sections. Firstly, a brief introduction to the topic, the proposed methodology, and the problem that the authors want to solve. After, in the Methodology section, the authors offer a detailed explanation of the solution presented. Next, the Case Study, where the characteristics of the dataset used as input, focusing on the community and the active consumers. In the Results and 
Discussion section, an analysis of the findings regarding the global perspective as well as the point of view of five selected consumers.

\section{Methodology}

In this section, the proposed methodology framework and assumptions are introduced. Since the focus is to optimally manage the participants in the DR event, the mathematical formulation respects the optimization scheduling to minimize the Aggregator's costs due to flexibility provision. By managing the availability of the local community, the entity manager will be able to mitigate limit violations by triggering DR events to reduce the tension on the faulty node. Figure 1 presents the proposed methodology.

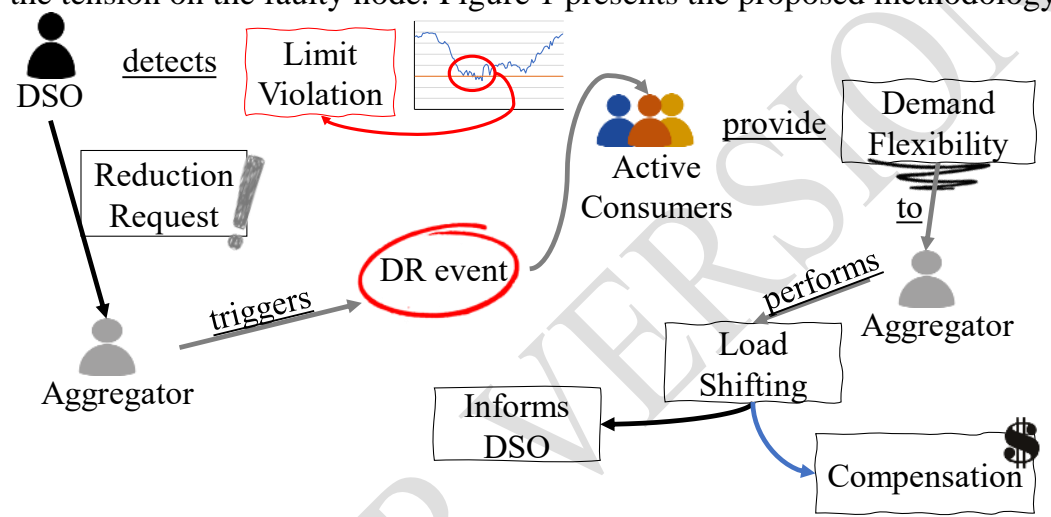

Fig. 1. Proposed Methodology

Performed the power transit, as soon as the limit violation is detected, the DR event is triggered, and the participants are requested to reduce the contracted amount. It must be noticed that active consumers register to offer the flexibility voluntarily for the participation and receive compensation if there is a change in their baseline profile. The availability from the demand side will be crucial. The consumer has power over the appliances - even resorting to smart equipment, the user has always the freedom of choice. So, it is important to find ways to deal with the uncertainty. For instance, give the right compensation and reduce the discomfort that may incur from the event. In this way, the authors design the tool to aid the Aggregator in this complex task, being the focus of the proposed methodology. Triggered the DR event, the Scheduling phase starts. A linear approach is employed to optimally manage the smart city and the local community since can deal with several types of active small resources: consumers participating in DR programs (PDR), DG units (PDG), and even the joint of these two concepts. The DG is considered a priority in this approach but, when the amount is reduced, external suppliers are also considered. Two types of external suppliers were integrated: regular (PSUPR) and additional (PSUPA), considering the quantities contracted at distinct prices and where the last is only used in extreme cases (more expensive). Let $p$ be the number of DG units, $c$ the number of consumers, sr the number of 
regular suppliers, sa the number of additional suppliers, and the actual period. The objective function is represented in Equation 1 [14].

Min. $\mathrm{OF}=\sum\left[\mathrm{P}_{\mathrm{DG}}(\mathrm{p}, \mathrm{t}) \mathrm{C}_{\mathrm{DG}}(\mathrm{p}, \mathrm{t})\right]+\sum\left[\mathrm{P}_{\mathrm{DR}}(\mathrm{c}, \mathrm{t}) \mathrm{C}_{\mathrm{DR}}(\mathrm{c}, \mathrm{t})\right]+\sum\left[\mathrm{P}_{\mathrm{SUPA}}(\mathrm{sa}, \mathrm{t})\right.$

$\left.\mathrm{C}_{\text {SUPA }}(\mathrm{sa}, \mathrm{t})\right]+\sum\left[\mathrm{P}_{\text {SUPR }}(\mathrm{sr}, \mathrm{t}) \mathrm{C}_{\text {SUPR }}(\mathrm{sr}, \mathrm{t})\right]+\mathrm{P}_{\text {NSP }}(\mathrm{t}) \mathrm{C}_{\mathrm{NSP}}(\mathrm{t})$

The network balance constraint is presented in Equation 2, achieving an equilibrium between consumption and generation where the sum of the requested reduction to the consumer initial load ( $\left.\mathrm{P}_{\text {initialLoad }}\right)$ should equal the total generation from $\mathrm{DG}$ units and external suppliers. In the extreme eventuality of demand not met by generation means, the variable from Non-Supplied Power $\left(\mathrm{P}_{\mathrm{NSP}}\right)$ was added. The network is being well managed if the parameter PNSP is zero.

$\sum\left[\mathrm{P}^{\text {initial }}(\mathrm{c}, \mathrm{t})-\mathrm{P}_{\mathrm{DR}}(\mathrm{c}, \mathrm{t})\right]=\sum\left[\mathrm{P}_{\mathrm{DG}}(\mathrm{p}, \mathrm{t})\right]+\sum\left[\mathrm{P}_{\text {SUPA }}(\mathrm{sa}, \mathrm{t})\right]+\sum\left[\mathrm{P}_{\text {SUPR }}(\mathrm{sr}, \mathrm{t})\right]$ $+\mathrm{P}_{\mathrm{NSP}}(\mathrm{t})$

The remaining constraints are presented between Equation 3 and Equation 10. In Equation 3, the constraint for the maximum value of reduction for each consumer contracted flexibility.

$$
\mathrm{P}_{\mathrm{DR}}(\mathrm{c}, \mathrm{t}) \leq \mathrm{P}_{\mathrm{DR}}{ }^{\mathrm{Max}}(\mathrm{c}, \mathrm{t})
$$

Equation 4 to Equation 6 describe DG units restricting upper, lower bounds and a total maximum value of generation.

$$
\begin{aligned}
& P_{D G}(p, t) \leq P_{D G}{ }^{M a x}(p, t) \\
& P_{D G}(p, t) \geq P_{D G}{ }^{M i n}(p, t) \\
& \sum\left[P_{D G}(p, t)\right] \leq P_{D G}{ }^{\text {Total }}(t)
\end{aligned}
$$

Equation 7 to Equation 10 refer to External Suppliers restricting them superiorly and total amount, for each type.

$$
\begin{aligned}
& P_{\text {SUPR }}(s r, t) \leq P_{\text {SUPR }}{ }^{\text {Max }}(s r, t) \\
& \sum\left[P_{\text {SUPR }}(s r, t) \leq P_{\text {SUPR }}{ }^{\text {Total }}(t)\right. \\
& P_{\text {SUPA }}(s a, t) \leq P_{\text {SUPA }}{ }^{\text {Max }}(s a, t) \\
& \sum\left[P_{\text {SUPA }}(s a, t)\right] \leq P_{\text {SUPA }}{ }^{\text {Total }}(t)
\end{aligned}
$$

The following phase, Reduction Request, invite the active consumers to participate in the DR event and reduce the consumption to aid the Aggregator goal to mitigate the 
limit violation. As soon as the power transit is violation-free, the participants are remunerated with the proper compensation and the load is shifted to another period, convenient to both Aggregator and the active consumer reducing the discomfort caused.

\section{$3 \quad$ Case Study}

In this section, the information regarding the design of the case study is provided. The smart city has 96 consumers and, the grid is composed of 234 buses. The day is divided into periods of 15 minutes, so 96 periods were considered. On periods 38, 40, 42, 43, and 44 occurred voltage limit violation, as can be seen in Figure 2. To solve the problem, DR programs are applied however only 64 consumers were willing to participate in DR events.

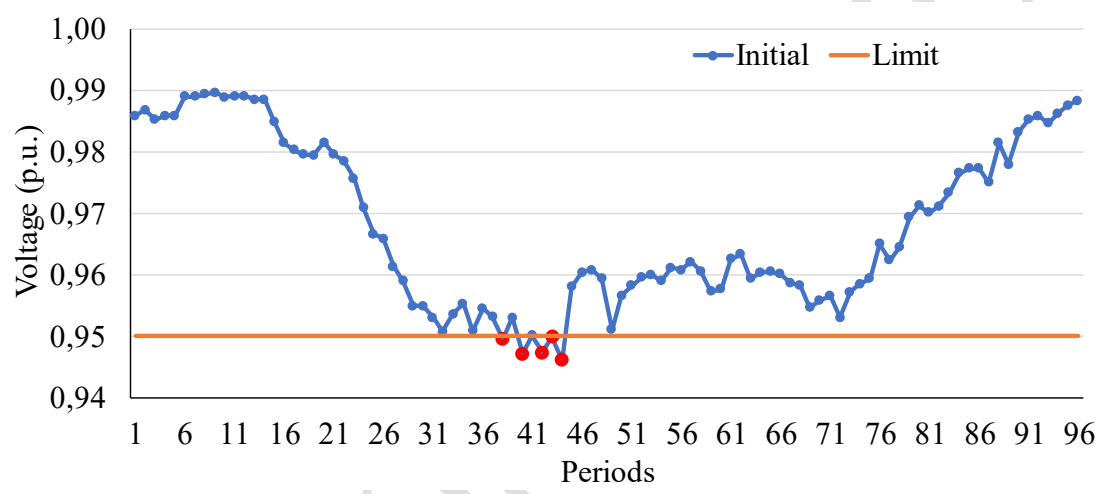

Fig. 2. Limit Violation Detection

The main goal of the present paper is assessment the limit from the proposed methodology when performing load shifting to mitigate threshold violation. Load shifting is applied to mitigate limit violation throughout the day - the programed consumption is moved forward in time to another period without causing more complications. It was assumed that shifted consumption should be scheduled between 5 hours after the DR event but never 24 hours after the same - a range of 19 hours.

The DR event is trigged upon limit violation detection and participants should reduce the amount contracted at the time requested to be further scheduled according to the mentioned range. In this study, the availability of the consumer at the DR event time was also considered.

To avoid additional violations upon the event, the shifted load is assigned to different periods according to the consumer needs - preventing any discomfort. The remuneration is a monetary value of $0.22 \mathrm{~m} . \mathrm{u} . / \mathrm{kW}$. Understanding the impact on microgrids and five selected consumers, the next section will present and discuss the results. The chosen consumers are from different parts of the grid -3 of them are in the same node as the limit violation and the remaining are from another distant part. 


\section{$4 \quad$ Results and Discussion}

Throughout the present section, the results of the proposed methodology to properly manage the smart city by the Aggregator perspective - in this case, mitigate the limit violations. Firstly, from a global perspective, Figure 3 shows the comparison between initial and final consumption after the application of the proposed method. The discrepancies between both curves are noticeable in the periods where the limits violations were detected - flexibility provided by active consumers.



Fig. 3. Load Shifting

With the reduction, the voltage values were restored to their proper place. As can be seen in Figure 4, not only the values are above the limit, but further limit violations were created with this solution. Since the appliances from active consumers were shifted to different hours - according to their availability and to reduce the discomfort cause - the final curve follows the same line as the initial in the remaining periods.



Fig. 4. Global perspective

From a more specific perspective, the five different consumers are confronted by the impact on the baseline profile as well as the remuneration granted from their participation. From the microgrid perspective, both Consumer 1 and 2 have their location far from the crisis point. Figure 5 and Figure 8 show the comparison between initial and 
final consumption as well as the events in which the active consumer in question participate.

It is also highlighted where the consumptions were reallocated in the same days. May occur reallocations to the day after, according to the consumers' convenience. Also, the authors consider the willingness to participate in a DR event according to a certain context - period, since different consumers have different habits which should be considered when creating a solution for DR events.

First, Figure 5 shows the results from Consumer 1, which participate in only one of the five events. The consumption was shifted from period 44 to period 83 .

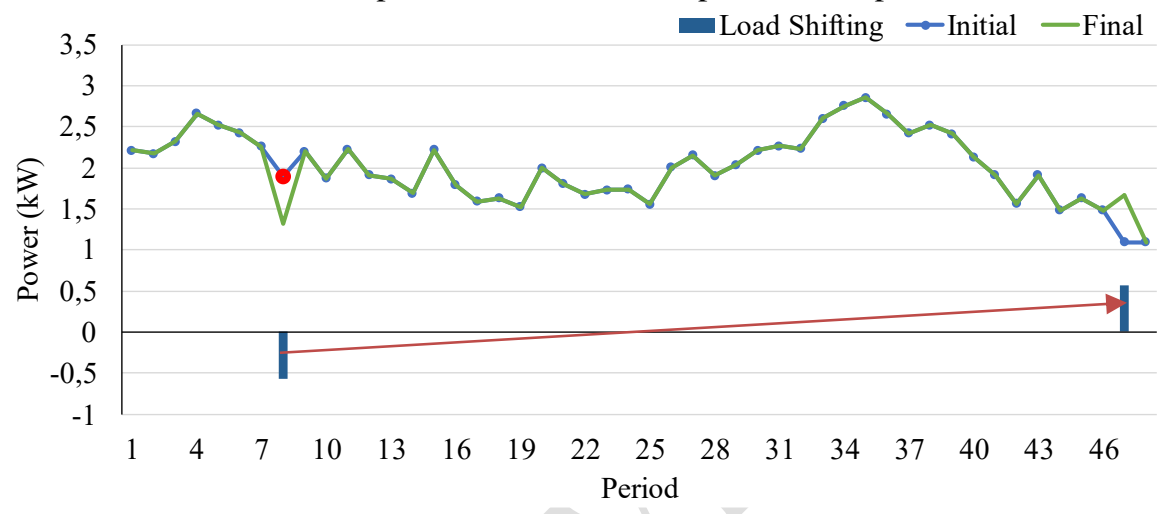

Fig. 5. Comparison between initial and final load profile - Consumer 1.

Regarding Consumer 2, Figure 6 shows that participated in four of five events, although only three were scheduled for this day with high values of flexibility - between 2 and $2.5 \mathrm{~kW}$.

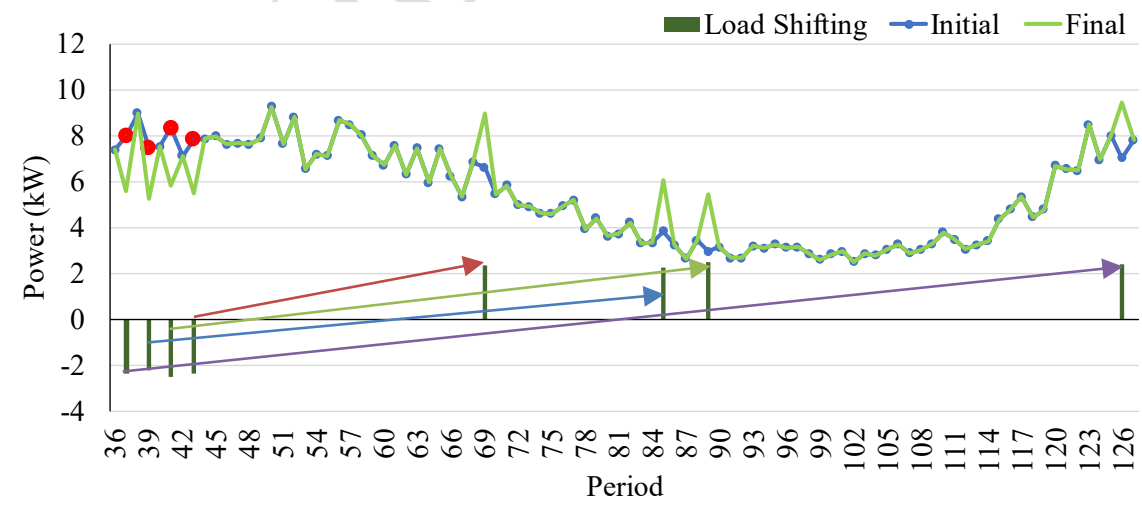

Fig. 6. Comparison between initial and final load profile - Consumer 2.

Consumer 3, Consumer 4, and Consumer 5 are from the same node where the limit violation was detected. Figure 7 presents the comparison between the initial and final load profiles from Consumer 3. This participant only takes part in two of five of the 
events which only one was scheduled to later that day. However, was the one, from the five selected that had a higher amount of reduction - almost $3 \mathrm{~kW}$ on period 40 .

In respect to Consumer 4, although DR participant, the results show that this active consumer does not contribute to any event, as can be seen in Figure 8. As referred earlier, participation is voluntary and depends on the willingness and availability of the user.

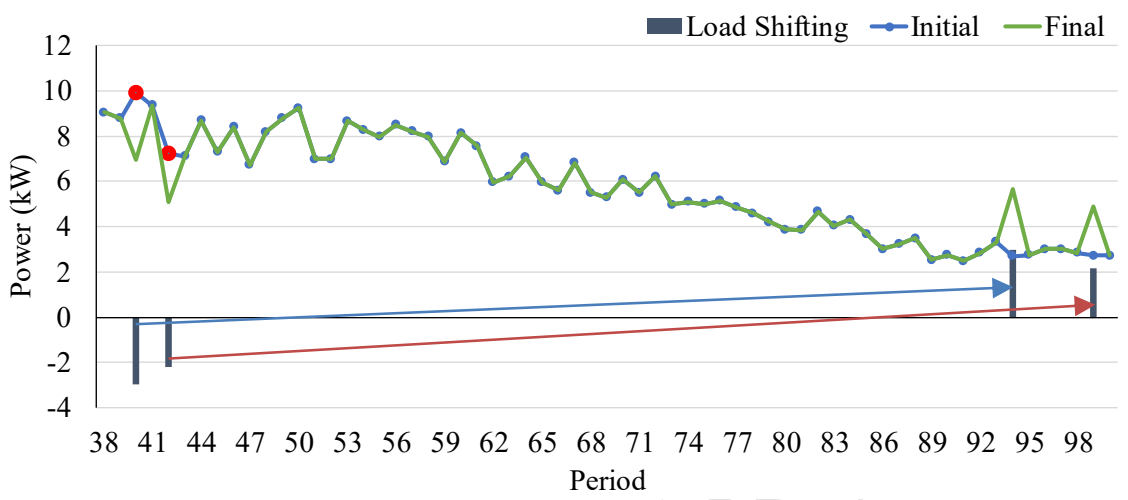

Fig. 7. Comparison between initial and final load profile - Consumer 3 .

This is one of the examples where the uncertainty on the response must also contemplate the context of which the event is inserted. Finally, Consumer 5 participate in four out of five events, as can be seen in Figure 8. Like Consumer 2, only three of the reductions were shifted to the same day.

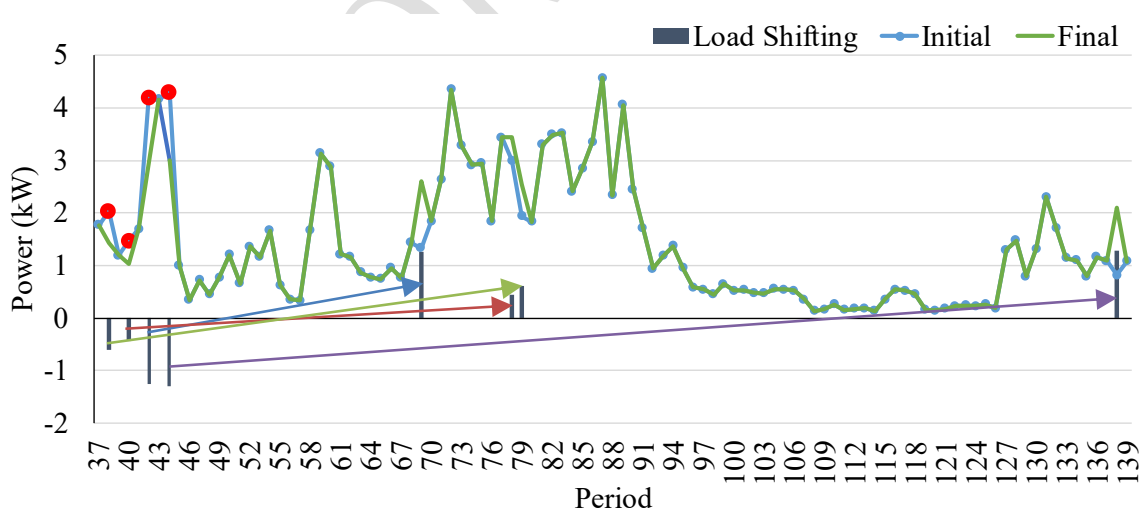

Fig. 8. Comparison between initial and final load profile - Consumer 5.

Table 1 presents the summary demonstrated earlier with the calculation of the remuneration received from each consumer in respect to their contribution to the management of the smart city. From this perspective, it is easier to see that none of the selected consumers participated in the mitigation of the limit violation of period 43 . In period 38 , only Consumer 2 and Consumer 5 provided flexibility with a total of $3 \mathrm{~kW}$. In period 
40 , three consumers contributed with $5.65 \mathrm{~kW}$ but were on period 42 where these consumers reduce more $-5.93 \mathrm{~kW}$. Finally, on period 44 , Consumer 1 , Consumer 2 , and Consumer 5 join the event and reduce a total of $4.21 \mathrm{~kW}$. Regarding the compensation, being the one with more contributions, Consumer 2 received 2.09 m.u. $/ \mathrm{kW}$ to shift the consumption to another period. After, Consumer 3 with 2.09 m.u. $/ \mathrm{kW}$, Consumer 5 with 0.79 m.u./kW and finally, Consumer 1 with 0.13 m.u. $/ \mathrm{kW}$

Table 1. Reduction per period and Remuneration values

\begin{tabular}{|c|c|c|c|c|c|c|}
\hline \multirow{2}{*}{$\begin{array}{c}\text { Consumer } \\
\text { ID }\end{array}$} & \multicolumn{5}{|c|}{ Reduction (kW) } & Remuneration \\
\cline { 2 - 6 } & 38 & 40 & 42 & 43 & 44 & (m.u./kW) \\
\hline $1-41$ & 0.00 & 0.00 & 0.00 & 0.00 & 0.57 & 0.13 \\
\hline $2-48$ & 2.39 & 2.24 & 2.50 & 0.00 & 2.35 & 2.09 \\
\hline $3-59$ & 0.00 & 2.97 & 2.17 & 0.00 & 0.00 & 1.13 \\
\hline $4-60$ & 0.00 & 0.00 & 0.00 & 0.00 & 0.00 & 0.00 \\
\hline $5-51$ & 0.61 & 0.44 & 1.26 & 0.00 & 1.29 & 0.79 \\
\hline
\end{tabular}

From the study presented in this paper, the following conclusions can be withdrawn:

- The Aggregator must have powerful tools and make the right assumptions when dealing with the uncertainty on DR events to mitigate other problems on the management of the microgrid.

- When applying load shifting techniques, consider not only the grid issues but also the discomfort from the consumer perspective.

- The active consumers must be motivated to continuously contribute to the management of the grid.

- Uncertain responses must be dealt with the right motivations - remember that participation is voluntary, and the consumer has freedom of choice, even though penalties may be applied.

The authors believe that develop solutions where the consumer behaviors are considered, namely regarding the context of the event, should be a step forward to implement DR programs in the real market.

\section{Conclusions}

The growing concern regarding the climate change impacts on the so-called normal functioning of the world motivates the energy sector to exchange non-renewable solutions for technologies more environmentally friendly. Distributed Generation has more popularity but, to the system, the unstable behavior may cause problems with the security and the reliability. Now, instead of the generation following the consumption requests, the demand side must contribute with more flexibility - empowering the consumer role in the energy market. In this way, the consumer concept is changing - have more information and power to control their consumption with signals given by the energy market. Resorting to the technologies, smart appliances ease this task. For instance, giving the control to a managing entity to schedule the appliance according to 
the user preferences. When DR events are triggered, according to the contract with the entity manager, it is expected a response from the active consumer, rewarded with the right compensation.

In the present paper, the authors test the proposed methodology in a scenario where a voltage limit violation was detected, and a DR event must be triggered to deal with the problem. From the results, the solution was capable of mitigating the violation and not cause other problems after. However, when analyzing five different consumers, was more evident the importance of context and motivation. The consumer must be available and willing to participate, without that, the DR event becomes a more complex problem due to the uncertainty associated.

In future works, the authors intend to further study the consumer behavior, the factors that impact and motivate to take some decisions to create the right tool, resorting to context-aware methods, to aid the Aggregator on its difficulty of managing the active communities.

\section{Acknowledgements}

This work has received funding from the European Union's Horizon 2020 research and innovation programme under project DOMINOES (grant agreement No 771066), from FEDER Funds through COMPETE program and from National Funds through (FCT) under the projects UIDB/00760/2020, COLORS (PTDC/EEI-EEE/28967/2017), CEECIND/02887/2017, and SFRH/BD/144200/2019, and from ANI (project GREEDi).

\section{References}

[1] M. Hu and F. Xiao, "Quantifying uncertainty in the aggregate energy flexibility of highrise residential building clusters considering stochastic occupancy and occupant behavior," Energy, vol. 194, p. 116838, Mar. 2020, doi: 10.1016/j.energy.2019.116838.

[2] S. Lokhande, V. P. Menon, and Y. K. Bichpuriya, "Modelling of demand response for utility's loac forecasting," in International Conference on the European Energy Market, EEM, Jun. 2017, pp. 1-6, doi: 10.1109/EEM.2017.7981985.

[3] S. Yu, F. Fang, Y. Liu, and J. Liu, "Uncertainties of virtual power plant: Problems and countermeasures," Appl. Energy, vol. 239, no. August 2018, pp. 454-470, Apr. 2019, doi: 10.1016/j.apenergy.2019.01.224.

[4] B. Wang, J. A. Camacho, G. M. Pulliam, A. H. Etemadi, and P. Dehghanian, "New reward and penalty scheme for electric distribution utilities employing load-based reliability indices," IET Gener. Transm. Distrib., vol. 12, no. 15, pp. 3647-3654, Aug. 2018, doi: 10.1049/iet-gtd.2017.1809.

[5] S. Tiwari, R. Sabzehgar, and M. Rasouli, "Load balancing in a microgrid with uncertain renewable resources and loads," in 2017 IEEE 8th International Symposium on Power Electronics for Distributed Generation Systems (PEDG), Apr. 2017, pp. 1-8, doi: 
10.1109/PEDG.2017.7972505.

[6] Z. A. Khan, D. Jayaweera, and H. Gunduz, "Smart meter data taxonomy for demand side management in smart grids," in 2016 International Conference on Probabilistic Methods Applied to Power Systems (PMAPS), Oct. 2016, pp. 1-8, doi: 10.1109/PMAPS.2016.7764143.

[7] B. Zeng, G. Wu, J. Wang, J. Zhang, and M. Zeng, "Impact of behavior-driven demand response on supply adequacy in smart distribution systems," Appl. Energy, vol. 202, pp. 125-137, Sep. 2017, doi: 10.1016/j.apenergy.2017.05.098.

[8] S. Chen and C. C. Liu, "From demand response to transactive energy: state of the art," J. Mod. Power Syst. Clean Energy, vol. 5, no. 1, pp. 10-19, 2017, doi: 10.1007/s40565016-0256-x.

[9] I. Ilieva, B. Bremdal, and S. Puranik, "Bringing Business and Societal Impact Together in an Evolving Energy Sector," Clean Energy Technol., vol. 7, no. 3, 2019, doi: 10.18178/jocet.2019.7.3.508.

[10] S. Zalzar and E. F. Bompard, "An Incentive-Based Settlement Mechanism for Participation of Flexible Demands in Day-ahead Markets," in 2019 International Conference on Smart Energy Systems and Technologies (SEST), Sep. 2019, pp. 1-6, doi: 10.1109/SEST.2019.8849038.

[11] Ö. Okur, N. Voulis, P. Heijnen, and Z. Lukszo, "Aggregator-mediated demand response: Minimizing imbalances caused by uncertainty of solar generation," Appl. Energy, vol. 247, no. October 2018, pp. 426-437, Aug. 2019, doi: 10.1016/j.apenergy.2019.04.035.

[12] C. Silva, P. Faria, and Z. Vale, "Demand response and distributed generation remuneration approach considering planning and operation stages," Energies, vol. 12, no. 14, p. 2721, 2019, doi: 10.3390/en12142722.

[13] J. M. Terras, C. Silva, Z. Vale, and P. Faria, "Consumers Modelling and Clustering for the Use of Flexibility to Mitigate Network Congestion," in CIRED 2020 Berlin Workshop, 2020, no. September, pp. 22-23.

[14] C. Silva, P. Faria, and Z. Vale, "Rating the Participation in Demand Response Programs for a More Accurate Aggregated Schedule of Consumers after Enrolment Period," Electronics, vol. 9, no. 2, p. 349, Feb. 2020, doi: 10.3390/electronics9020349. 